\title{
Multi-mode oscillations in classical Cepheids and RR Lyrae-type stars
}

\author{
Paweł Moskalik \\ Copernicus Astronomical Centre, ul. Bartycka 18, 00-716 Warsaw, Poland \\ email: pam@camk.edu.pl
}

\begin{abstract}
I review different types of multi-mode pulsations observed in classical Cepheids and in RR Lyrae-type stars. The presentation concentrates on the newest results, with special emphasis on recently detected nonradial oscillations.
\end{abstract}

Keywords. stars: oscillations, stars: variables: Cepheids, stars: variables: RR Lyr

\section{Introduction}

Most of classical (Pop. I) Cepheids and RR Lyrae-type stars are periodic, single-mode radial pulsators. The long-term lightcurve modulation, observed in some RR Lyrae-type variables, was for many years the only known exception from this simple picture. This phenomenon, known as the Blazhko effect, was discovered a century ago (Blazhko 1907, Shapley 1916) and still lacks a satisfactory explanation. Current understanding of the Blazhko modulation has been reviewed by Kovács (2009) and by Szabó (these proceedings) and will not be discussed here. Instead, I will focus on various forms of multi-mode pulsations, i.e. pulsations with several different oscillation modes simultaneously excited.

\section{Classical double-mode radial pulsators}

F+1O double-mode Cepheids. The first double-mode pulsators, UTrA and TU Cas, were identified more than fifty years ago (Oosterhoff 1957a,b). These two Cepheids pulsate simultaneously in the two lowest radial modes - the fundamental mode (F) and the first overtone (1O). Double-mode Cepheids of this class have period ratios of $P_{1} / P_{0}=0.694-0.746$. Almost 200 such stars are currently known in the Galaxy and the Magellanic Clouds (Soszyński et al. 2008b, 2010a, 2011b, 2012, Marquette et al. 2009, Smolec \& Moskalik 2010 and references therein). A few have also been discovered in M33 (Beaulieu et al. 2006) and most recently in M31 (Poleski 2013a).

$F+1 O$ double-mode $R R$ Lyrae-type stars (RRd stars). These variables are RR Lyraetype analogs of the $\mathrm{F}+1 \mathrm{O}$ double-mode Cepheids. AQ Leo, the prototype of the class, was discovered by Jerzykiewicz \& Wenzel (1977). Since then, nearly 2000 RR Lyrae stars of this type have been identified. They are observed in the Galactic field (Wils 2010, Poleski 2013b), in many Galactic globular clusters (e.g. Walker 1994, Walker \& Nemec 1996, Corwin et al. 2008), in the Magellanic Clouds (Soszyński et al. 2009, 2010b, 2012) and in nearby dwarf spheroidal galaxies (e.g. Cseresnjes 2001, Clementini et al. 2006, Bernard et al. 2009). In most stellar populations, their period ratios are in a very narrow range of $P_{1} / P_{0}=0.742-0.748$. The only exception is the Galactic Bulge, where variables with $P_{1} / P_{0}$ as low as 0.726 are found (Soszyński et al. 2011a). These low period ratios indicate higher metallicities, up to $Z \sim 0.008$.

$1 O+2 O$ double-mode Cepheids. Another type of double-mode pulsations was detected a few years later in the short period Cepheid CO Aur (Mantegazza 1983). In this star 
the period ratio is very different, $P_{2} / P_{1}=0.801$, implying pulsations in the first $(1 \mathrm{O})$ and the second (2O) radial overtones. Currently, about 500 double-mode Cepheids of this type are known, most in the Magellanic Clouds (Soszyński et al. 2008b, 2010a, 2012, Marquette et al. 2009). Only 19 are identified in the Galaxy (Smolec \& Moskalik 2010 and references therein; Soszyński et al. 2011b). One such variable has also been found in the spiral galaxy M31 (Poleski 2013a).

\section{New types of multi-mode radial pulsators}

In the next 20 years following the discovery of CO Aur, no new types of multi-mode radial pulsators were identified. This started to change only with the advent of microlensing surveys, which collected photometric data for thousands of Cepheids and RR Lyraetype stars. Such data allowed finding rare forms of oscillations. In just a few years, the inventory of multi-mode radial pulsators was enlarged by several new classes. Further discoveries came from analysis of ultra-precise photometry obtained with Kepler and CoRoT planet-hunting space telescopes.

$1 O+3 O$ double-mode Cepheids. Cepheids pulsating in the first (1O) and the third (3O) radial overtones are extremely rare. Only two examples are known, both in the Large Magellanic Cloud (hereafter LMC, Soszyński et al. 2008a). Both variables have period ratio of $P_{3} / P_{1}=0.677$. The amplitude of the third overtone is very low, in both cases below $0.03 \mathrm{mag}$ in the $I$-band.

$F+2 O$ double-mode RR Lyrae-type stars. This type of pulsation is also very rare and so far has been detected only in 9 stars (see Moskalik 2013 and references therein). All but one have been discovered with photometry obtained from space. The ratio of the two pulsation periods falls in a very narrow range of $P_{2} / P_{0}=0.582-0.593$, implying pulsations in the radial fundamental mode $(\mathrm{F})$ and the second radial overtone $(2 \mathrm{O})$. In most stars of this class the fundamental mode is modulated, i.e. displays a Blazhko effect. However, this is not a rule. In two variables with the longest pulsation periods (V350 Lyr and KIC 7021124) the amplitudes are constant.

Triple-mode Cepheids. The first two variables of this type were identified by Moskalik et al. (2004). Currently, only 10 triple-mode Cepheids are known in both Magellanic Clouds and in the Galaxy (Soszyński et al. 2008a, 2010a, 2011b). They can be divided into two classes: those which pulsate in the fundamental mode and the first two radial overtones $(\mathrm{F}+1 \mathrm{O}+2 \mathrm{O}$ type; 4 objects $)$ and those which pulsate in the first three radial overtones $(1 \mathrm{O}+2 \mathrm{O}+3 \mathrm{O}$ type; 6 objects). Properties of all known triple-mode Cepheids are summarized in Table 1 . These rare objects are very important and interesting targets for asteroseismology. Modeling of their three pulsation periods strongly constrains parameters of these stars and, consequently, imposes tight constraints on Cepheid evolutionary tracks (Moskalik \& Dziembowski 2005).

Blazhko effect in 1O+2O double-mode Cepheids. Long-term periodic amplitude modulation was detected in many $1 \mathrm{O}+2 \mathrm{O}$ double-mode Cepheids of the LMC (Moskalik et al. 2004, 2006, Moskalik \& Kołaczkowski 2009). This behaviour is quite common and occurs in at least $20 \%$ (but possibly in as many as 35\%) of these stars. Both overtones vary with the common period $\left(P_{B}>700 \mathrm{~d}\right)$, but variations are anticorrelated - the maximum amplitude of one mode coincides with minimum amplitude of the other. The phases (or equivalently periods) of both modes are modulated, too. The phenomenon closely resembles the Blazhko effect in the RR Lyrae-type stars. The only difference is that not one, but two radial modes are being modulated. Within the accuracy of the data, this modulation is periodic, and the modulation period is the same for both overtones. 
Table 1. Triple-mode Cepheids.

\begin{tabular}{|c|c|c|c|c|c|c|c|c|}
\hline star & $P_{0}[$ day $]$ & $P_{1}[$ day $]$ & $P_{2}[$ day $]$ & $P_{3}[$ day $]$ & $P_{1} / P_{0}$ & $P_{2} / P_{1}$ & $P_{3} / P_{2}$ & ref. \\
\hline \multicolumn{9}{|c|}{$1 \mathrm{O}+2 \mathrm{O}+3 \mathrm{O}$ Cepheids } \\
\hline OGLE-LMC-CEP- 1847 & & 0.5795 & 0.4666 & 0.3921 & & 0.8052 & 0.8404 & 1,2 \\
\hline OGLE-LMC-CEP- 2147 & & 0.5413 & 0.4360 & 0.3663 & & 0.8056 & 0.8401 & 1,2 \\
\hline OGLE-LMC-CEP-3025 & & 0.5687 & 0.4582 & 0.3850 & & 0.8057 & 0.8402 & 2 \\
\hline OGLE-SMC-CEP-3867 & & 0.2688 & 0.2174 & 0.1824 & & 0.8086 & 0.8392 & 3 \\
\hline OGLE-BLG-CEP-16 & & 0.2955 & 0.2340 & 0.1951 & & 0.7919 & 0.8337 & 4 \\
\hline OGLE-BLG-CEP-30 & & 0.2304 & 0.1830 & 0.1522 & & 0.7943 & 0.8316 & 4 \\
\hline \multicolumn{9}{|c|}{$\mathrm{F}+1 \mathrm{O}+2 \mathrm{O}$ Cepheids } \\
\hline OGLE-LMC-CEP-0857 & 1.4631 & 1.0591 & 0.8552 & & 0.7239 & 0.8075 & & 2 \\
\hline OGLE-LMC-CEP-1378 & 0.5150 & 0.3849 & 0.3094 & & 0.7474 & 0.8037 & & 2 \\
\hline OGLE-SMC-CEP-1077 & 0.8973 & 0.6565 & 0.5289 & & 0.7316 & 0.8056 & & 3 \\
\hline OGLE-SMC-CEP-1350 & 0.9049 & 0.6660 & 0.5379 & & 0.7360 & 0.8076 & & 3 \\
\hline
\end{tabular}

References: 1 - Moskalik et al. (2004), 2 - Soszyński et al. (2008a), 3 - Soszyński et al. (2010a), 4 Soszyński et al. (2011b).
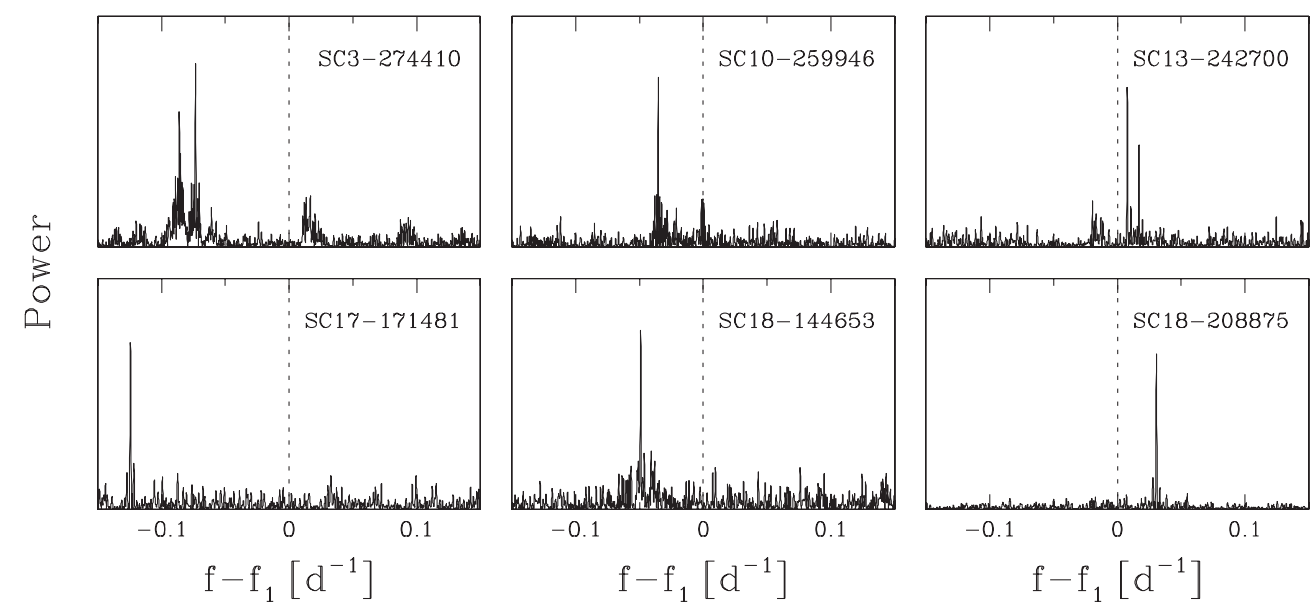

Figure 1. Nonradial modes in LMC first-overtone Cepheids. Prewhitened frequency spectra after subtracting the dominant radial mode (indicated with a dashed line) are displayed.

\section{Nonradial modes in Cepheids and RR Lyrae-type stars}

Excitation of nonradial modes is very common in pulsating stars. Modes of this type are observed in all classes of main-sequence pulsators (Gautschy \& Saio 1996) as well as in oscillating white dwarfs (Winget \& Kepler 2008, Fontaine, these proceedings), subdwarfs (Randall, these proceedings) and in stochastically driven subgiants and red giants (Hekker, these proceedings). But classical pulsating variables - Cepheids and RR Lyraetype stars - seemed to be different. They seemed to avoid excitation of any nonradial oscillations at all. This picture has changed only in the last decade. New observations prove that nonradial modes are present also in the classical pulsators.

\subsection{Nonradial modes in LMC Cepheids}

The first convincing detection of nonradial modes in classical Cepheids came with the analysis of LMC photometry of the OGLE-II survey (Moskalik et al. 2004, Moskalik \& Kołaczkowski 2008, 2009). In 37 first overtone Cepheids, low amplitude secondary frequencies have been found in close proximity to the dominant radial mode $(\Delta f<$ $\left.0.125 \mathrm{~d}^{-1}\right)$. Six of these variables are displayed in Fig. 1. Similar secondary frequencies 


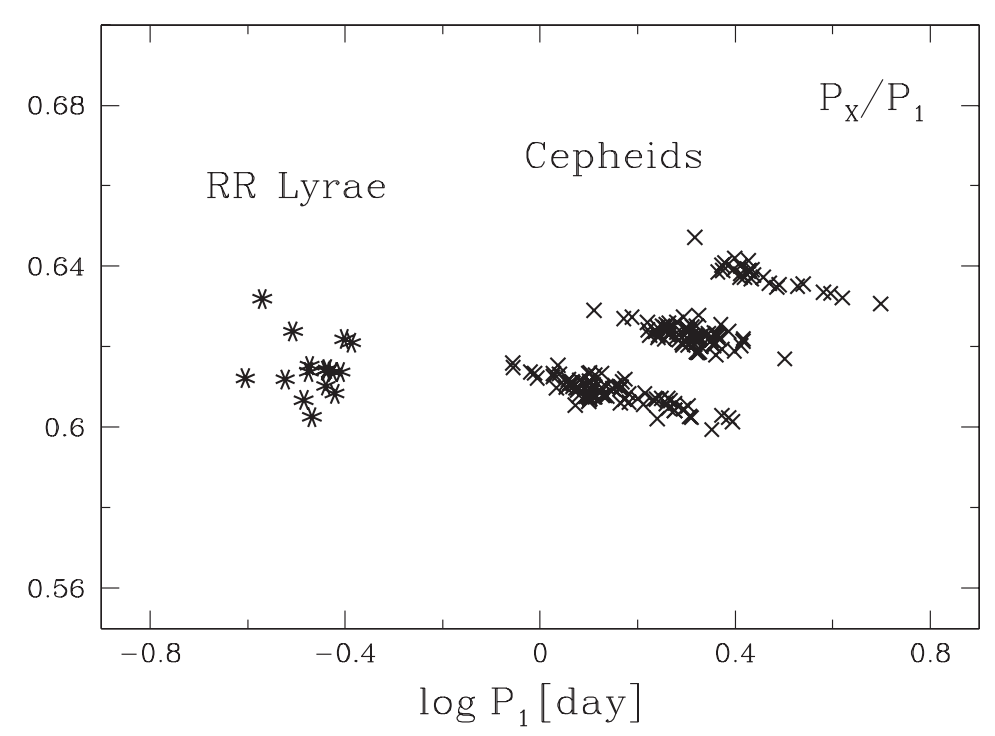

Figure 2. Petersen diagram for Cepheids and RR Lyrae-type stars with period ratio of $P_{X} / P_{1}=0.60-0.64$.

have also been detected in two $\mathrm{F}+1 \mathrm{O}$ double-mode Cepheids, where they are found close to the first radial overtone. In most cases only one secondary peak is present, but sometimes two peaks are seen. In the latter case, both peaks always appear on the same side of the radial mode. The observed frequency pattern cannot be explained by any form of modulation of the radial mode. Modulation should always produce an equally spaced frequency multiplet centered on the primary peak, and such a structure is not found in any of these stars. Because secondary frequencies are too close to the frequency of the radial first overtone, they cannot be explained by any radial mode, either. Consequently, they must correspond to nonradial modes of oscillations.

\subsection{Double-mode pulsators with period ratios of $0.60-0.64$}

Cepheids. In some Magellanic Cloud Cepheids a different type of multiperiodicity is found - a single secondary mode is detected with a frequency much above that of the radial mode. The ratio of the secondary and the primary period falls in a narrow range of $P_{X} / P_{1}=0.60-0.64$. The first eight Cepheids of this class have been discovered in the LMC by Moskalik \& Kołaczkowski (2008). Currently, more than 170 such pulsators are known in both Magellanic Clouds (Soszyński et al. 2008b, 2010a, Moskalik \& Kołaczkowski 2009). The phenomenon is restricted to the first overtone Cepheids and $\mathrm{F}+1 \mathrm{O}$ double-mode Cepheids (only one star). When plotted on the period ratio vs. period diagram (so called Petersen diagram), these variables form three very tight parallel sequences (see Fig. 2). They are as tight as in case of the radial double-mode pulsators. However, none of the sequences fits theoretical prediction for the two radial modes (Dziembowski \& Smolec 2009, Dziembowski 2012). This implies that the secondary mode is nonradial. Considering that this mode has to be unstable, the theoretical analysis suggests that it must be an f-mode of high angular degree of $l=42-50$ (Dziembowski 2012).

$R R$ Lyrae-type stars. Secondary modes with the period ratio of $0.60-0.64$ are found in RR Lyrae-type stars as well. 16 such variables have been identified so far, including four stars observed by the Kepler space telescope (Moskalik et al. 2013, 2014 and references 


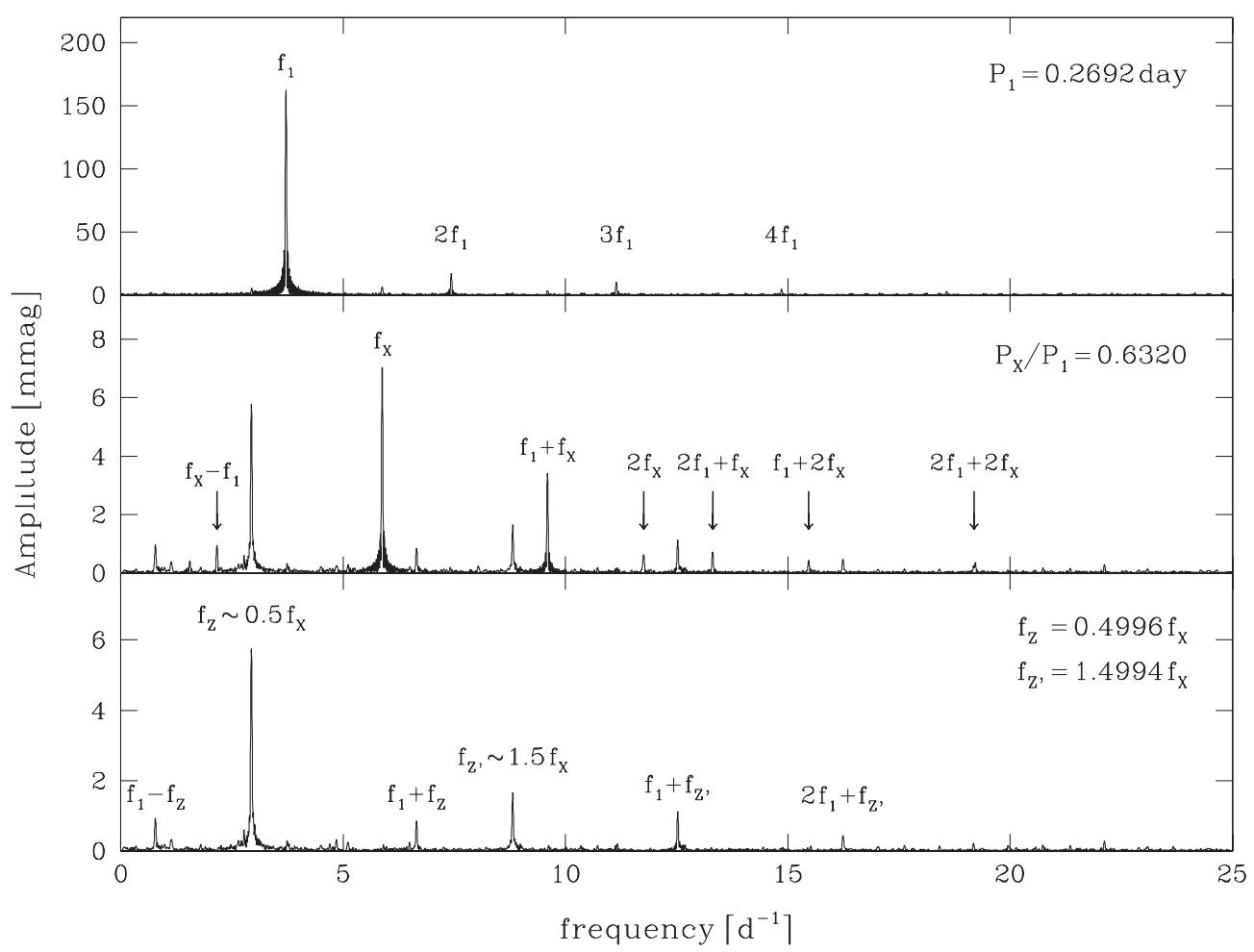

Figure 3. Prewhitening sequence for KIC 5520878. Upper panel: frequency spectrum of original data. Middle panel: frequency spectrum after subtracting $f_{1}$ and its harmonics. Bottom panel: frequency spectrum after subtracting $f_{1}, f_{X}$ and their harmonics and linear combinations.

therein). Most of these variables are dominated by the first radial overtone (RRc stars), but two are double-mode pulsators (RRd stars). The amplitude of the secondary mode is always extremely low, in the mmag range. Interestingly, this mode is found in all four RRc stars observed by Kepler. This suggests that such double-mode pulsations are rather common and should be detected in many more RRc variables, provided high-quality photometry is available. Just like in the case of Cepheids, comparison of observed period ratios with theoretical models implies that the secondary frequency must correspond to a nonradial mode (Moskalik et al. 2014).

The RR Lyrae-type stars displaying the puzzling period ratio of $0.60-0.64$ are very similar to their Cepheid siblings. In both cases this phenomenon occurs only in the firstovertone pulsators and in the $\mathrm{F}+1 \mathrm{O}$ double-mode pulsators. The period ratios fall in the same narrow range. This is shown in Fig. 2, where we plot both groups of variables on a common Petersen diagram. The only difference between the two groups is that classical Cepheids split into three separate sequences, whereas the RR Lyrae-type stars do not. Finally, in both types of stars the secondary mode is nonradial. These similarities lead to the conclusion that Cepheids and RR Lyrae-type stars with the period ratio of $P_{X} / P_{1}=0.60-0.64$ form a very homogenous group, constituting a new, well defined class of multimode pulsators.

Period doubling of the secondary mode in RR Lyrae-type stars. In Fig. 3 we display a prewhitening sequence for KIC 5520878, one of the RRc stars in the Kepler field (Moskalik et al. 2014). After subtracting the dominant radial pulsation $\left(f_{1}\right)$ and its harmonics, the 


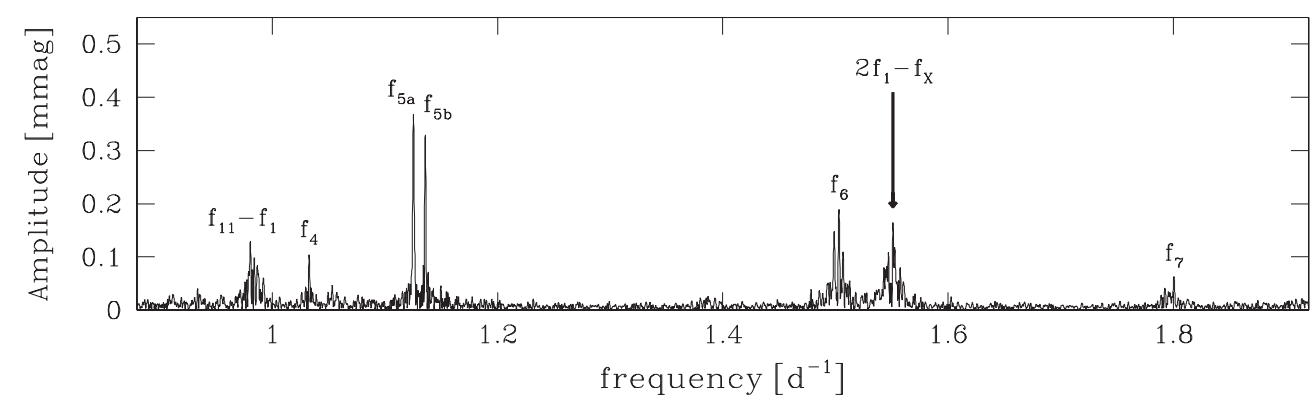

Figure 4. Low frequency low amplitude modes in KIC 5520878. Fourier transform of Q1-Q10 lightcurve is computed after prewhitening the data of the dominant frequency $f_{1}, f_{X}$, their harmonics, subharmonics and 5 highest combination peaks. Nonradial modes appear at $f_{4}$, $f_{5}$ (doublet), $f_{6}$ (triplet) and $f_{7} . f_{11}-f_{1}$ is a combination peak associated with another low amplitude mode. The frequency of the radial fundamental mode is estimated to be $f_{0} \sim 2.75 \mathrm{~d}^{-1}$.

frequency spectrum of the residuals (middle panel) reveals a secondary frequency at $f_{X}=$ $5.879 \mathrm{~d}^{-1}$, which yields a period ratio of $P_{X} / P_{1}=0.632$. A harmonic of $f_{X}$ and several linear combinations with $f_{1}$ are also present. But ultra-precise Kepler photometry reveals even more. After prewhitening the data with $f_{1}, f_{X}$ and their harmonic and combination frequencies (bottom panel), the highest residual peak appears at $f_{Z}=2.937 \mathrm{~d}^{-1}$, that is at $\sim f_{X} / 2$. In other words, $f_{Z}$ is not an independent frequency, but a subharmonic of $f_{X}$. Another subharmonic is found at $f_{Z^{\prime}} \sim(3 / 2) f_{X}$. The detection of subharmonic frequencies is highly significant. Their presence is a characteristic signature of a period doubling of the secondary mode, $f_{X}$. The same signature of the period doubling behaviour is seen in all four RRc variables in the Kepler field (Moskalik et al. 2013, 2014). It is also detected in the RRd variable AQ Leo (Gruberbauer et al. 2007). Thus, RR Lyrae-type stars with the period ratio of $0.60-0.64$ are yet another class of pulsators in which period doubling can occur. We recall, that this phenomenon has recently been discovered in two other classes of pulsating variables - in Blazhko RRab stars (Szabó et al. 2010) and in the BL Herculis-type stars (Smolec et al. 2012). Its origin can be traced to a half-integer resonance between the pulsation modes (Moskalik \& Buchler 1990).

\subsection{Other nonradial modes in RRc stars}

With the benefit of high-quality Kepler data it is possible to search for modes which are even weaker than $f_{X}$ and its subharmonic peaks. Such modes are discovered in three Kepler RRc variables (Moskalik et al. 2014). All but one of these oscillations have amplitudes below $0.4 \mathrm{mmag}$. The majority of them generate combination peaks with the dominant radial pulsation $\left(f_{1}\right)$, which proves that they indeed originate in the RRc star and do not come from blending with another variable.

The richest harvest of low amplitude modes is found in KIC 5520878, where 15 such oscillations are detected. Based on the period ratios, one of these modes can be identified with the second radial overtone, but all others must be nonradial. Interestingly, several modes have frequencies significantly lower than the radial fundamental mode (see Fig. 4). This implies that these nonradial oscillations are not $p$-modes, but must be of either gravity or mixed mode character. Low-frequency modes of this type are discovered also in the other two RRc stars.

\subsection{Nonradial modes in RRab stars}

Low-amplitude modes other than the radial second overtone are detected in six RRab variables. They are listed in Table 2 . For each secondary mode, instead of its period, we 
Table 2. Secondary periodicities in RRab stars

\begin{tabular}{lcccl}
\hline star & $P_{0}[$ day $]$ & $P / P_{0}$ & $P / P_{0}$ & ref. \\
\hline V1127 Aql & 0.3560 & 0.7271 & 0.6966 & 1,2 \\
RR Lyr & 0.5669 & 0.7557 & & 5 \\
V354 Lyr & 0.5617 & 0.7295 & 0.8555 & 3 \\
V360 Lyr & 0.5576 & 0.7210 & & 3 \\
V445 Lyr & 0.5131 & 0.7306 & 0.7031 & 4 \\
CoRoT 105288363 & 0.5674 & 0.7216 & 0.7764 & 4 \\
& & 0.7407 & & \\
\hline
\end{tabular}

References: 1 - Chadid et al. (2010), 2 - Poretti et al. (2010), 3 - Benkő et al. (2010), 4 - Guggenberger et al. (2012), 5 - Molnár et al. (2012).

provide the period ratio of this mode and the dominant fundamental radial pulsation, $P / P_{0}$. The period ratios given in the column 4 of the Table are clearly incompatible with those of the radial modes. Therefore, these secondary oscillations must be nonradial. The situation is somewhat more complicated for modes listed in column 3 . Their period ratios are more or less similar to that observed in the RRd stars. On this ground, the secondary modes of column 3 are usually identified with the radial first overtone. However, the double-mode RR Lyrae-type stars do not populate the $P / P_{0}$ vs. $P_{0}$ diagram in a random way. Instead, they form a rather narrow, well defined sequence on this diagram (Soszyński et al. 2009, 2011a). Putting this differently, there is an empirical relation between the period ratio and the period. It is easy to check that V1127 Aql is the only variable of Table 2 which follows this relation. For all the remaining variables, the secondary modes listed in column 3 deviate considerably from the empirical trend. Therefore, identification of these modes with the radial first overtone should be treated with great caution. These periodicities most likely correspond to nonradial modes, the frequencies of which are just close to that of the radial overtone. We note, that a small number of similar outliers from the empirical $P / P_{0}$ vs. $P_{0}$ relation have also been identified in the LMC and in the Galactic bulge (Soszyński et al. 2009, 2011a). The secondary periods in those objects might correspond to nonradial modes, too.

The tendency of nonradial oscillations to appear preferentially around the frequency of the radial overtone is not a surprise. In fact, it is consistent with the theory. Linear nonadiabatic calculations show that nonradial modes can be excited in the RR Lyraetype stars, and their growth rates are highest in the vicinity of the radial modes (Van Hoolst et al. 1998, Dziembowski \& Cassisi 1999). The growth rates reach maximum values at frequencies slightly above that of the radial mode. This is true both for modes of $l=1,2$ and for the strongly trapped modes (STU modes) of higher spherical degrees. Thus, nonradial oscillations which are most likely to be excited should yield period ratios somewhat lower than the radial first overtone. This prediction is in good agreement with observational picture summarized in Table 2 .

\section{Acknowledgements}

This research is supported by the Polish National Science Centre through grant DEC2012/05/B/ST9/03932. Generous IAU support in the form of a travel grant is also acknowledged.

\section{References}

Beaulieu, J.-P., Buchler, J. R., Marquette, J.-B., et al. 2006, ApJ, 653, L101

Benkő, J. M., Kolenberg, K., Szabó, R., et al. 2010, MNRAS, 409, 1585

Bernard, E. J., Monelli, M., Gallart, C., et al. 2009, ApJ, 699, 1742 
Blazhko, S. 1907, AN, 175, 325

Chadid, M., Benkő, J. M., Szabó, R., et al. 2010, A\&A, 510, A39

Clementini, G., Greco, C., Held, E. V., et al. 2006, MemSAIt, 77, 249

Corwin, T. M., Borissova, J., Stetson, P. B., et al. 2008, AJ, 135, 1459

Cseresnjes, P. 2001, A\&BA, 375, 909

Dziembowski, W. A. 2012, AcA, 62, 323

Dziembowski, W. A. \& Cassisi, S. 1999, AcA, 49, 371.

Dziembowski, W. A. \& Smolec, R. 2009, AIP-CP, 1170, 83

Gautschy, A. \& Saio, H. 1996, ARAA, 34, 551

Gruberbauer, M., Kolenberg, K., Rowe, J. F., et al. 2007, MNRAS, 379, 1498

Guggenberger, E., Kolenberg, K., Nemec, J. M., et al. 2012, MNRAS, 424, 649

Jerzykiewicz, M. \& Wenzel, W. 1977, AcA, 27, 35

Kovács, G. 2009, AIP-CP, 1170, 261

Mantegazza, L. 1983, A\&A, 118, 321

Marquette, J.-B., Beaulieu, J.-P., Buchler, J. R., et al. 2009, A\&A, 495, 249

Molnár, L., Kolláth, Z., Szabó, R. et al. 2012, ApJ, 757, L13

Moskalik, P. 2013, in: J. C. Suárez, R. Garrido, L. A. Balona, \& J. Christensen-Dalsgaard (eds.), Stellar Pulsations: Impact of New Instrumentation and New Insights, Astrophysics and Space Sci. Proc., Vol. 31 (Berlin, Heidelberg: Springer-Verlag), p. 103

Moskalik, P. \& Buchler, J. R. 1990, ApJ, 355, 590

Moskalik, P. \& Dziembowski, W. A. 2005, A\& $A, 434,1077$

Moskalik, P. \& Kołaczkowski, Z. 2008, CoAst, 157, 343

Moskalik, P. \& Kołaczkowski, Z. 2009, MNRAS, 394, 1649

Moskalik, P., Kolenberg, K., Smolec, R., et al. 2014, MNRAS, to be submitted

Moskalik, P., Kołaczkowski, Z., \& Mizerski, T. 2004, ASP-CS, 310, 498

Moskalik, P., Kołaczkowski, Z., \& Mizerski, T. 2006, MemSAIt, 77, 563

Moskalik, P., Smolec, R., Kolenberg, K., et al. 2013, in: J. C. Suárez, R. Garrido, L. A. Balona, \& J. Christensen-Dalsgaard (eds.), Stellar Pulsations: Impact of New Instrumentation and New Insights, Astrophysics and Space Sci. Proc., Vol. 31 (Berlin, Heidelberg: SpringerVerlag), Poster No. 34 (arXiv: 1208.4251)

Oosterhoff, P. T. h. 1957a, BAN, 13, 317

Oosterhoff, P. T. h. 1957b, BAN, 13, 320

Poleski, R. 2013a, ApJ, 778, 147

Poleski, R. 2013b, A\&A, submitted (arXiv: 1309.1168)

Poretti, E., Paparó, M., Deleuil, M., et al. 2010, A\&AA, 520, A108

Shapley, H. 1916, ApJ, 43, 217

Smolec, R. \& Moskalik, P. 2010, A\&A A, 524, A40

Smolec, R., Soszyński, I., Moskalik, P., et al. 2012, MNRAS, 419, 2407

Soszyński, I., Dziembowski, W. A., Udalski, A., et al. 2011a, AcA, 61, 1

Soszyński, I., Poleski, R., Udalski, A., et al. 2008a, AcA, 58, 153

Soszyński, I., Poleski, R., Udalski, A., et al. 2008b, AcA, 58, 163

Soszyński, I., Poleski, R., Udalski, A., et al. 2010a, AcA, 60, 17

Soszyński, I., Udalski, A., Pietrukowicz, P., et al. 2011b, AcA, 61, 285

Soszyński, I., Udalski, A., Poleski, R., et al. 2012, AcA, 62, 219

Soszyński, I., Udalski, A., Szymański, M. K., et al. 2009, AcA, 59, 1

Soszyński, I., Udalski, A., Szymański, M. K., et al. 2010b, AcA, 60, 165

Szabó, R., Kolláth, Z., Molnár, L., et al. 2010, MNRAS, 409, 1244

Van Hoolst, T., Dziembowski, W. A., \& Kawaler, S. D. 1998, MNRAS, 297, 536

Walker, A. R. 1994, AJ, 108, 555

Walker, A. R. \& Nemec, J. M. 1996, AJ, 112, 2026

Wils, P. 2010, IBVS, 5955

Winget, D. E. \& Kepler, S. O. 2008, ARAA, 46, 157 